\title{
COMPLEX SPACE FORMS IMMERSED IN COMPLEX SPACE FORMS
}

\author{
BY
}

H. NAKAGAWA AND K. OGIUE(1)

\begin{abstract}
We determine all the isometric immersions of complex space forms into complex space forms. Our result can be considered as the local version of a well-known result of Calabi.
\end{abstract}

A Kaehler manifold of constant holomorphic curvature is called a complex space form. By a Kaehler submanifold we mean a complex submanifold with the induced Kaehler metric. E. Calabi [1] gave a classification of Kaehler imbeddings of complete and simply connected complex space forms into complete and simply connected complex space forms. The local version of Calabi's result has been conjectured to be true by the second author [4] and he gave some partial solutions [2], [3].

The purpose of this paper is to prove the following two theorems which furnish the complete solutions to the conjecture. Throughout this paper we denote by $M_{n}(c)$ an $n$-dimensional complex space form of constant holomorphic curvature $c$.

THEOREM 1. Let $M_{n}(c)$ be a Kaehler submanifold immersed in $M_{n+p}(\tilde{c})$. If $\tilde{c}>0$ and the immersion is full, then $\tilde{c}=\nu c$ and $n+p=\left(\begin{array}{c}n+\nu \\ \nu\end{array}\right)-1$ for some positive integer $\nu$.

THEOREM 2. Let $M_{n}(c)$ be a Kaehler submanifold immersed in $M_{n+p}(\tilde{c})$. If $\tilde{c} \leqslant 0$, then $\tilde{c}=c$ (i.e., $M_{n}(c)$ is totally geodesic in $M_{n+p}(\tilde{c})$ ).

1. Kaehler submanifolds in $M_{n+p}(\tilde{c})$. Let $M$ be an $n$-dimensional Kaehler submanifold immersed in $M_{n+p}(\tilde{c})$. We choose a local field of unitary frames $e_{1}, \ldots, e_{n}, e_{n+1}, \ldots, e_{n+p}$ in $M_{n+p}(\tilde{c})$ in such a way that, restricted to $M$,

Received by the editors October 16, 1973.

AMS (MOS) subject classifications (1970). Primary 53B25, 53B35.

Key words and phrases. Complex space form, Kaehler submanifold.

(1)The first author's research was done under the program "Sonderforschungsbereich Theoretische Mathematik" at the University of Bonn.

(2)Throughout this paper we use the following convention on the range of indices unless otherwise stated:
$A, B, C, \ldots=1, \ldots, n, n+1, \ldots, n+p$,
$i, j, k, \ldots=1, \ldots, n$,
$\alpha, \beta, \gamma, \ldots=n+1, \ldots, n+p$. 
$e_{1}, \ldots, e_{n}$ are tangent to $M$. Let $\omega^{1}, \ldots, \omega^{n}, \omega^{n+1}, \ldots, \omega^{n+p}$ be the field of dual frames. Then the Kaehler metric $\widetilde{g}$ of $M_{n+p}(\tilde{c})$ is given by $\tilde{g}=$ $\Sigma_{A} \omega^{A} \bar{\omega}^{A}$ and the structure equations of $M_{n+p}(\tilde{c})$ are given by $\left({ }^{2}\right)$

$$
d \omega^{A}+\sum_{B} \omega_{B}^{A} \wedge \omega^{B}=0, \quad \omega_{B}^{A}+\bar{\omega}_{A}^{B}=0,
$$

$$
d \omega_{B}^{A}+\sum_{C} \omega_{C}^{A} \wedge \omega_{B}^{C}=\widetilde{\Omega}_{B}^{A}, \quad \widetilde{\Omega}_{B}^{A}=\sum_{C, D} \widetilde{R}_{B C \bar{D}^{A}}^{A} \wedge \bar{\omega}^{D}
$$

Since $M_{n+p}(\widetilde{c})$ is a complex space form of constant holomorphic curvature $\widetilde{c}$, we have

$$
\widetilde{R}_{B C \bar{D}}^{A}=\frac{\widetilde{c}}{4}\left(\delta_{B}^{A} \delta_{C D}+\delta_{C}^{A} \delta_{B D}\right)
$$

Restricting these forms to $M$, we have

$$
\omega^{\alpha}=0
$$

and the Kaehler metric $g$ of $M$ is given by $g=\Sigma_{i} \omega^{i} \bar{\omega}^{i}$.

It follows from (1.1), (1.4) and Cartan's lemma that we may write

$$
\omega_{i}^{\alpha}=\sum_{j} h_{i j}^{\alpha} \omega^{j}, \quad h_{i j}^{\alpha}=h_{j i}^{\alpha}
$$

Moreover we obtain

$$
\begin{array}{ll}
d \omega^{i}+\sum_{j} \omega_{j}^{i} \wedge \omega^{j}=0, & \omega_{j}^{i}+\bar{\omega}_{i}^{j}=0, \\
d \omega_{j}^{i}+\sum_{k} \omega_{k}^{i} \wedge \omega_{j}^{k}=\Omega_{j}^{i}, & \Omega_{j}^{i}=\sum_{k, l} R_{j k l}^{i} T \omega^{k} \wedge \bar{\omega}^{l}, \\
d \omega_{\beta}^{\alpha}+\sum_{\gamma} \omega_{\gamma}^{\alpha} \wedge \omega_{\beta}^{\gamma}=\Omega_{\beta}^{\alpha}, & \Omega_{\beta}^{\alpha}=\sum_{k, l} R_{\beta k T}^{\alpha} \omega^{k} \wedge \bar{\omega}^{l} .
\end{array}
$$

From (1.2), (1.3), (1.4), (1.5), and (1.7), we have

$$
\Omega_{j}^{i}=\sum_{k, l}\left\{\frac{\widetilde{c}}{4}\left(\delta_{j}^{i} \delta_{k l}+\delta_{k}^{i} \delta_{j l}\right)-\sum_{\alpha} h_{j k}^{\alpha} \bar{h}_{i l}^{\alpha}\right\} \omega^{k} \wedge \bar{\omega}^{l} .
$$

Similarly from (1.2), (1.3), (1.4), (1.5), and (1.8), we have

$$
\Omega_{\beta}^{\alpha}=\sum_{k, l}\left(\frac{\widetilde{c}}{4} \delta_{\beta}^{\alpha} \delta_{k l}+\sum_{j} h_{j k}^{\alpha} \bar{h}_{j l}^{\beta}\right) \omega^{k} \wedge \bar{\omega}^{l} .
$$

If we define $h_{i j k}^{\alpha}$ and $h_{i j \bar{k}}^{\alpha}$ by

$$
\sum_{k} h_{i j k}^{\alpha} \omega^{k}+\sum_{k} h_{i j \bar{k}}^{\alpha} \bar{\omega}^{k}=d h_{i j}^{\alpha}-\sum_{k} h_{k j}^{\alpha} \omega_{i}^{k}-\sum_{k} h_{i k}^{\alpha} \omega_{j}^{k}+\sum_{\beta} h_{i j}^{\beta} \omega_{\beta}^{\alpha},
$$

then we can easily see

$$
h_{i j k}^{\alpha}=h_{i k j}^{\alpha}, \quad h_{i j \bar{k}}^{\alpha}=0
$$


We may define inductively the successive derivatives of $h_{i j}^{\alpha}$ by

$$
\left\{\begin{array}{l}
\sum_{l} h_{i_{1} \cdots i_{k} l}^{\alpha} \omega^{l}+\sum_{l} h_{i_{1} \cdots i_{k}}^{\alpha} \bar{\omega}^{l} \\
=d h_{i_{1} \cdots i_{k}}^{\alpha}-\sum_{r=1}^{k} \sum_{j_{r}} h_{i_{1} \cdots j_{r} \cdots i_{k}}^{\alpha} \omega_{i_{r}}^{j_{r}}+\sum_{\beta} h_{i_{1} \cdots i_{k}}^{\beta} \omega_{\beta}^{\alpha}, \\
\sum_{m} h_{i_{1} \cdots i_{k}}^{\alpha}{ }^{l m} \omega^{m}+\sum_{m} h_{i_{1} \cdots i_{k} \bar{l} \bar{m}}^{\alpha} \bar{\omega}^{m} \\
=d h_{i_{1} \cdots i_{k}}^{\alpha}-\sum_{r=1}^{k} \sum_{j_{r}} h_{i_{1} \cdots j_{r} \cdots i_{k}}^{\alpha} \omega_{i_{r}}^{j_{r}} \\
-\sum_{m} h_{i_{1} \cdots i_{k}}^{\alpha} \bar{\omega}_{l}^{m}+\sum_{\beta} h_{i_{1} \cdots i_{k}}^{\beta} \omega_{\beta}^{\alpha} .
\end{array}\right.
$$

If $h_{i_{1} \ldots i_{k}}^{\alpha}$ is symmetric with respect to all indices, then taking the exterior derivative of the first equality of (1.13) and using (1.6), (1.7), and (1.8), we get

$$
\begin{aligned}
\sum_{l, m} h_{i_{1} \cdots i_{k} l m}^{\alpha} \omega^{l} \wedge \omega^{m}-\sum_{l, m}\left(h_{i_{1} \cdots i_{k}}^{\alpha} \overline{l m}^{m}-h_{i_{1} \cdots i_{k}}^{\alpha}\right) \omega^{l} \wedge \bar{\omega}^{m} \\
\quad+\sum_{l, m} h_{i_{1} \cdots i_{k}}^{\alpha} \overline{l m} \bar{\omega}^{l} \wedge \bar{\omega}^{m} \\
=-\sum_{r=1}^{k} \sum_{i_{r}} h_{i_{1} \cdots j_{r} \cdots i_{k}}^{\alpha} \Omega_{i_{r}}^{i_{r}}+\sum_{\beta} h_{i_{1} \cdots i_{k}}^{\beta} \Omega_{\beta}^{\alpha},
\end{aligned}
$$

from which, together with (1.9) and (1.10), we obtain

$$
\begin{aligned}
& h_{i_{1} \cdots i_{k} l m}^{\alpha}=h_{i_{1} \cdots i_{k} m l}^{\alpha}, \quad h_{i_{1} \cdots i_{k}}^{\alpha} \overline{l m}=h_{i_{1} \cdots i_{k} \bar{m} \bar{l}}^{\alpha}, \\
& h_{i_{1} \cdots i_{k} l \bar{m}}^{\alpha}-h_{i_{1} \cdots i_{k} \overline{m l}}^{\alpha} \\
& =\frac{\tilde{c}}{4}\left\{(k-1) h_{i_{1} \cdots i_{k}}^{\alpha} \delta_{l m}+\sum_{r=1}^{k} h_{i_{1} \cdots l \cdots i_{k}}^{\alpha} \delta_{i_{r} m}\right\} \\
& \quad-\sum_{r=1}^{k} \sum_{i_{r} \beta} h_{i_{1} \cdots i_{r} \cdots i_{k}}^{\alpha} h_{i_{r}}^{\beta} \bar{h}_{i_{r} m}^{\beta}-\sum_{j_{, \beta} \beta} h_{i_{1} \cdots i_{k}}^{\beta} h_{j l}^{\alpha} \bar{h}_{j m}^{\beta} .
\end{aligned}
$$

Since $h_{i j}^{\alpha}$ and $h_{i j k}^{\alpha}$ are symmetric with respect to all indices, (1.14) implies inductively that

$$
h_{i_{1} \cdots i_{k}}^{\alpha} \text { is symmetric with respect to all indices, }
$$

and consequently (1.15) holds for all integers $k \geqslant 2$. 
2. Proof of theorems. Since $M_{n}(c)$ is a complex space form of constant holomorphic curvature $c$, the curvature forms $\Omega_{j}^{i}$ are given by

$$
\Omega_{j}^{l}=\sum_{k, l} R_{j k \bar{l}}^{l} \omega^{k} \wedge \bar{\omega}^{l}=\frac{c}{4} \sum_{k, l}\left(\delta_{j}^{i} \delta_{k l}+\delta_{k}^{i} \delta_{j l}\right) \omega^{k} \wedge \bar{\omega}^{l},
$$

which, together with (1.9), implies

$$
\sum_{\alpha} h_{i k}^{\alpha} \bar{h}_{j l}^{\alpha}=\frac{\tilde{c}-c}{4}\left(\delta_{i j} \delta_{k l}+\delta_{i l} \delta_{j k}\right)
$$

From (2.1) and the second property of (1.12), we obtain

$$
\sum_{\alpha} h_{i_{1} \cdots i_{k}}^{\alpha} \bar{h}_{j l}^{\alpha}=0 \text { for } k \geqslant 3 \text {. }
$$

Substituting (2.1) and (2.2) into (1.15), we have

$$
\begin{aligned}
h_{i_{1} \cdots i_{k} l \bar{m}}^{\alpha}= & h_{i_{1} \cdots i_{k} \overline{m l}}^{\alpha}-\frac{\tilde{c}-k c}{4} h_{i_{1} \cdots i_{k}}^{\alpha} \delta_{l m} \\
& +\frac{c}{4} \sum_{r=1}^{k} h_{i_{1} \cdots l \cdots i_{k}}^{\alpha} \delta_{i_{r} m} \text { for } k \geqslant 3,
\end{aligned}
$$

and, in particular, applying this relation to the first term of the right-hand side repeatedly and taking account of (1.12), we can obtain

$$
h_{i_{1} \cdots i_{k} \tau}^{\alpha}=-\frac{\tilde{c}-(k-1) c}{4} \sum_{r=1}^{k} h_{i_{1} \cdots \hat{i}_{r} \cdots i_{k}}^{\alpha} \delta_{i_{r} l}
$$

where the notation ` means the omission of the index $i_{r}$. Since $h_{i j k}^{\alpha}$ is symmetric, from (2.1), (2.2), and (2.3), we have

$$
\begin{aligned}
h_{i j k}^{\alpha} \bar{h}_{l m n}^{\alpha}=\frac{(\tilde{c}-c)(\tilde{c}-2 c)}{4^{2}}\left(\delta_{i l} \delta_{j m} \delta_{k n}\right. & +\delta_{i l} \delta_{j n} \delta_{k m}+\delta_{i m} \delta_{j n} \delta_{k l} \\
& \left.+\delta_{i m} \delta_{j l} \delta_{k n}+\delta_{i n} \delta_{j l} \delta_{k m}+\delta_{i n} \delta_{j m} \delta_{k l}\right)
\end{aligned}
$$

First of all, as a generalization of (2.1) and (2.4), we shall prove the following.

LEMMA 1.

$$
\sum_{\alpha} h_{i_{1} \cdots i_{k}}^{\alpha} \bar{h}_{j_{1} \cdots j_{l}}^{\alpha}=\left\{\begin{array}{l}
0 \text { for } k \neq l, \\
\frac{1}{4^{k-1}} \prod_{r=1}^{k-1}(c-r c) \sum_{\sigma} \delta_{\sigma\left(i_{1}\right) j_{1}} \cdots \delta_{\sigma\left(i_{k}\right) j_{k}} \\
\quad \text { for } k=l,
\end{array}\right.
$$


where $\Sigma_{\sigma}$ is the summation on all permutations with respect to indices $i_{1}, \ldots, i_{k}$.

Proof. We shall prove the second equality by induction. The cases where $k=2$, and $k=3$ reduce to (2.1) and (2.4) respectively.

We suppose that the following equalities hold:

$$
\sum_{\alpha} h_{i_{1} \cdots i_{l}}^{\alpha} \bar{h}_{j_{1} \cdots j_{l}}^{\alpha}=\frac{1}{4^{l-1}} \prod_{r=1}^{l-1}(\tilde{c}-r c) \sum_{\sigma} \delta_{\sigma\left(i_{1}\right) j_{1}} \cdots \delta_{\sigma\left(i_{l}\right) j_{l}}
$$

for $l \leqslant k$.

Then it follows from (2.3) that

$$
\begin{aligned}
\sum_{\alpha} h_{i_{1} \cdots i_{k}}^{\alpha} \bar{h}_{j_{1} \cdots j_{k} m}^{\alpha}=-\sum_{\alpha} h_{i_{1} \cdots i_{k}}^{\alpha} \bar{m} \bar{h}_{j_{1} \cdots j_{k}}^{\alpha} \equiv 0 \\
\left(\bmod \sum_{\alpha} h_{l_{1} \cdots l_{k-1}}^{\alpha} \bar{h}_{m_{1} \cdots m_{k}}^{\alpha} \delta_{i j}\right) .
\end{aligned}
$$

Making use of (2.3) and the supposition (2.6) of the induction repeatedly, we see that

$$
\sum_{\alpha} h_{i_{1} \cdots i_{k}}^{\alpha} \bar{h}_{i_{1} \cdots j_{k} m}^{\alpha}=0 \text {, i.e., } \sum_{\alpha} h_{i_{1} \cdots i_{k} m^{m}}^{\alpha} \bar{h}_{j_{1} \cdots j_{k}}^{\alpha}=0
$$

From (2.3) and the second equality of (2.7), we have

$$
\begin{aligned}
\sum_{\alpha} h_{i_{1} \cdots i_{k+1}}^{\alpha} \bar{h}_{j_{1} \cdots j_{k+1}}^{\alpha} & \\
= & \frac{1}{4^{k}} \prod_{r=1}^{k}(\tilde{c}-r c) \sum_{\sigma} \delta_{\sigma\left(i_{1}\right) j_{1}} \cdots \delta_{\sigma\left(i_{k+1}\right) j_{k+1}} .
\end{aligned}
$$

This shows that (2.5) holds for any integer $k$.

By the similar argument, we can prove the first equality of (2.5) noting that we may assume $k>l$ without loss of generality. Q.E.D.

From the second equality of (2.5) we have

$$
\begin{aligned}
\sum_{\alpha} \sum h_{i_{1} \cdots i_{k+1}}^{\alpha} \bar{h}_{i_{1} \cdots i_{k+1}}^{\alpha} & \\
& =\frac{1}{4^{k}} n(n+1) \cdots(n+k)(\tilde{c}-c)(\tilde{c}-2 c) \cdots(\tilde{c}-k c) .
\end{aligned}
$$

LEMMA 2. If $c>0$, then $\tilde{c}=v c$ for some positive integer $\nu$.

PRoof. Suppose that there exists no integer $l$ such that $\tilde{c}=l c$. Then, since $c>0$ so that $\tilde{c}>c>0$, there exists an integer $k$ satisfying $(k-1) c<\widetilde{c}$ $<k c$. For such $k$, the right-hand side of $(2.8)$ is negative, which contradicts the fact that the left-hand side is nonnegative. Q.E.D. 
Next we prove the following.

LEMMA 3. (1) If $\tilde{c}=\nu c$ for a positive integer $\nu$, then $n+p \geqslant\left(\begin{array}{c}n+\nu \\ \nu\end{array}\right)-1$. (2) If $\tilde{c}>0 \geqslant c$ or $0 \geqslant \tilde{c}>c$, then $n+p \geqslant\left(\begin{array}{c}n+\nu \\ \nu\end{array}\right)-1$ for any positive integer $\nu$.

Proof. Put $m_{l}=\left(\begin{array}{c}n+l \\ l\end{array}\right)-(n+1)$ and suppose $p<m_{\nu}$. Let $H=\left(H_{\mu}^{\lambda}\right)$ be a matrix of order $m_{\nu}$ defined by

$$
\begin{cases}H_{\mu}^{\alpha-n}=\left\{\begin{array}{cc}
h_{i_{1} i_{2}}^{\alpha} & \text { for } \mu \leqslant m_{2}, \\
h_{i_{1} i_{2} i_{3}}^{\alpha} & \text { for } m_{2}<\mu \leqslant m_{3} \\
\cdots & \\
h_{i_{1} \cdots i_{\nu}}^{\alpha} & \text { for } m_{\nu-1}<\mu \leqslant m_{\nu}
\end{array}\right. \\
H_{\mu}^{\lambda}=0 & \text { for } p<\lambda \leqslant m_{\nu} .\end{cases}
$$

Then it follows from (2.5) that

$$
{ }^{t} \bar{H} H=\left[\begin{array}{ccccc}
2 ! \frac{\tilde{c}-c}{4} & & & & \\
& \cdot & & & \\
& & * & & \\
& & \cdot & \\
& & & & \nu ! \frac{\Pi_{r=1}^{\nu-1}(\tilde{c}-r)}{4^{\nu-1}}
\end{array}\right],
$$

where

$$
*=\frac{1}{4^{j-1}}\left(r_{1} !+r_{2} !+\cdots+r_{l} !\right) \prod_{s=1}^{j-1}(c-s c)
$$

and $r_{1}+\cdots+r_{l}=j$. The assumption on $c$ and $\tilde{c}$ implies that the determinant of $H$ is not zero, which contradicts the definition of the matrix $H$. Q.E.D.

Theorem 2 follows immediately from Lemma 3.

In order to complete the proof of Theorem 1, it suffices to show the following.

LEMMA 4. If $\tilde{c}=\nu c$ for a positive integer $\nu$ and the immersion is full, then $n+p=\left(\begin{array}{c}n+\nu \\ \nu\end{array}\right)-1$.

Proof. In consideration of Lemma 3(1), we suppose $n+p>\left(\begin{array}{c}n+\nu \\ \nu\end{array}\right)-1$. 
Let $A_{2}=\left(A_{2 \beta}^{\alpha}\right)$ be a matrix of order $p$ defined by $A_{2 \beta}^{\alpha}=\Sigma_{k, l} h_{k l}^{\alpha} \bar{h}_{k l}^{\beta}$. It is easily seen that $A_{2}$ is a Hermitian matrix. Furthermore, we consider a $p \times\left(\begin{array}{c}n+1 \\ 2\end{array}\right)$ matrix $H_{2}=\left(h_{i j}^{\alpha}\right)$. Then these two matrices satisfy

$$
A_{2} H_{2}=\frac{\tilde{c}-c}{2} H_{2}, \quad H_{2}{ }^{t} \bar{H}_{2}=A_{2}
$$

and the matrix ${ }^{t} \bar{H}_{2} H_{2}$ is nonsingular, which implies that the rank of the matrix $A_{2}$ is equal to $\left(\begin{array}{c}n+1 \\ 2\end{array}\right)$. We denote by $N_{x}$ the normal space to $M$ at $x$, and define a mapping $f_{2}$ of $N_{x} \times N_{x}$ into a complex field $C$ by

$$
f_{2}(X, Y)=\sum_{\alpha, \beta} A_{2 \beta}^{\alpha} \xi_{\alpha} \bar{\eta}_{\beta},
$$

where $X=\Sigma_{\alpha} \xi_{\alpha} e_{\alpha}$ and $Y=\Sigma_{\beta} \eta_{\beta} e_{\beta}$. Let $H_{p}$ be the set of all Hermitian matrices of order $p$, which is considered as a complex vector space. The unitary group $U(p)$ operates on $H_{p}$ as follows: For any Hermitian matrix $A \in H_{p}$ and any $U \in U(p), U(A)={ }^{t} \bar{U} A U$.

Since the matrix $A_{2}$ is invariant under $U(p)$, the mapping $f_{2}$ is well defined and it is a positive semidefinite Hermitian form of rank $r=\left(\begin{array}{c}n+1 \\ 2\end{array}\right)$ so that it can be normalized as

$$
f_{2}(X, X)=\lambda_{1}^{\prime} \xi_{n+1}^{\prime} \bar{\xi}_{n+1}^{\prime}+\cdots+\lambda_{r}^{\prime} \xi_{n+r}^{\prime} \bar{\xi}_{n+r}^{\prime}
$$

This means that we can choose a new unitary frame $\left(e_{i}, e_{\alpha_{1}}, e_{\beta}\right)$ at $x$ such that

$$
\omega_{i}^{\alpha_{1}} \neq 0, \quad \omega_{i}^{\beta}=0 \text { for }\left(\begin{array}{c}
n+1 \\
1
\end{array}\right) \leqslant \alpha_{1}<\left(\begin{array}{c}
n+2 \\
2
\end{array}\right), \beta \geqslant\left(\begin{array}{c}
n+2 \\
2
\end{array}\right) \text {. }
$$

Similarly, we can choose a new unitary frame $\left(e_{i}, e_{\alpha_{1}}, e_{\alpha_{2}}, e_{\beta}\right)$ such that

$$
\begin{aligned}
& \omega_{\alpha_{1}}^{\alpha_{2}} \neq 0, \quad \omega_{\alpha_{1}}^{\beta}=0 \\
& \text { for }\left(\begin{array}{c}
n+1 \\
1
\end{array}\right) \leqslant \alpha_{1}<\left(\begin{array}{c}
n+2 \\
2
\end{array}\right), \\
& \qquad\left(\begin{array}{c}
n+2 \\
2
\end{array}\right) \leqslant \alpha_{2}<\left(\begin{array}{c}
n+3 \\
3
\end{array}\right), \beta \geqslant\left(\begin{array}{c}
n+3 \\
3
\end{array}\right) .
\end{aligned}
$$

In fact, we take a unitary frame $\left(e_{i}, e_{\alpha_{1}}, e_{\beta}\right)$ satisfying property (2.9). Let $A_{3}=\left(A_{3 \beta}^{\alpha}\right)$ be a matrix of order $p$ defined by $A_{3 \beta}^{\alpha}=\Sigma_{i, j, k} h_{i j k}^{\alpha} \bar{h}_{i j k}^{\beta}$. Then $A_{3}$ is also a Hermitian matrix of $\operatorname{rank}\left({ }^{n+2} 3_{3}\right)$ and it is invariant under $U(p)$. This implies that a mapping $f_{3}$ of $N_{x} \times N_{x}$ into $C$ defined by

$$
f_{3}(X, Y)=\sum_{\alpha, \beta} A_{3 \beta}^{\alpha} \xi_{\alpha} \bar{\eta}_{\beta}
$$


where $X=\Sigma_{\alpha} \xi_{\alpha} e_{\alpha}$ and $Y=\Sigma_{\beta} \eta_{\beta} e_{\beta}$, is well defined and is also a positive semidefinite Hermitian form of $\operatorname{rank}\left({ }_{3}^{n+2}\right)$. Therefore we can choose a frame satisfying (2.10).

Since $h_{i_{1} \ldots i_{l}}^{\alpha}=0$ for $l \geqslant \nu+1$, we can inductively choose a unitary frame $\left(e_{i}, e_{\alpha_{1}}, \ldots, e_{\alpha_{\nu-1}}, e_{\beta}\right)$ at $x$ such that

$$
\begin{cases}\omega^{\beta}=0 & \text { for } \beta \geqslant\left(\begin{array}{c}
n+1 \\
1
\end{array}\right), \\
\omega_{i}^{\alpha} \neq 0, \quad \omega_{i}^{\beta}=0 & \text { for } \beta \geqslant\left(\begin{array}{c}
n+2 \\
2
\end{array}\right), \\
\vdots & \text { for } \beta \geqslant\left(\begin{array}{c}
n+r+1 \\
r+1
\end{array}\right), \\
\omega_{\alpha_{r-1}^{\alpha}}^{\alpha} \neq 0, \quad \omega_{\alpha_{r-1}}^{\beta}=0 & \\
\vdots & \\
\omega_{\alpha_{\nu-2}^{\alpha-1} \neq 0,}^{\alpha} \omega_{\alpha_{\nu-2}^{\beta}}^{\beta}=0 & \text { for } \beta \geqslant\left(\begin{array}{c}
n+v \\
v
\end{array}\right), \\
\omega_{\alpha_{\nu-1}^{\beta}}^{\beta}=0 & \text { for } \beta \geqslant\left(\begin{array}{c}
n+v \\
v
\end{array}\right),\end{cases}
$$

where $\left(\begin{array}{c}n+\eta \\ r\end{array}\right) \leqslant \alpha_{r}<\left(\begin{array}{c}n+r+1 \\ r+1\end{array}\right)$. Now we consider a distribution $\mathfrak{D l}$ on the frame bundle defined by

$$
\omega^{\beta}=0, \quad \omega_{i}^{\beta}=0, \quad \omega_{\alpha_{1}}^{\beta}=0, \ldots, \omega_{\alpha_{\nu-1}}^{\beta}=0 \text { for } \beta \geqslant\left(\begin{array}{c}
n+v \\
v
\end{array}\right),
$$

where $\left(\begin{array}{c}n+\eta \\ r\end{array}\right) \leqslant \alpha_{r}<\left(\begin{array}{c}n+r+1 \\ r+1\end{array}\right)$. Then it follows from the structure equations that

$$
\begin{aligned}
d \omega^{\beta} & =-\sum_{i=1}^{n} \omega_{i}^{\beta} \wedge \omega^{i}-\sum_{r=1}^{\nu-1} \sum_{\alpha_{r}} \omega_{\alpha_{r}}^{\beta} \wedge \omega^{\alpha_{r}}-\sum_{\gamma} \omega_{\gamma}^{\beta} \wedge \omega^{\gamma} \\
& \equiv 0\left(\bmod \omega^{\beta}, \omega_{i}^{\beta}, \omega_{\alpha_{1}}^{\beta}, \ldots, \omega_{\alpha_{\nu-1}^{\beta}}^{\beta}\right), \\
d \omega_{i}^{\beta} & =-\sum_{j=1}^{n} \omega_{j}^{\beta} \wedge \omega_{i}^{j}-\sum_{r=1}^{\nu-1} \sum_{\alpha_{r}} \omega_{\alpha_{r}}^{\beta} \wedge \omega_{i}^{\alpha_{r}}-\sum_{\gamma} \omega_{\gamma}^{\beta} \wedge \omega_{i}^{\gamma}+\widetilde{\Omega}_{i}^{\beta} \\
& \equiv 0\left(\bmod \omega^{\beta}, \omega_{i}^{\beta}, \omega_{\alpha_{1}}^{\beta}, \ldots, \omega_{\alpha_{\nu-1}^{\beta}}^{\beta}\right), \\
d \omega_{\alpha_{r}}^{\beta} & =-\sum_{j=1}^{n} \omega_{j}^{\beta} \wedge \omega_{\alpha_{r}}^{j}-\sum_{s=1}^{\nu-1} \sum_{\alpha_{s}} \omega_{\alpha_{s}}^{\beta} \wedge \omega_{\alpha_{r}}^{\alpha_{s}}-\sum_{\gamma} \omega_{\gamma}^{\beta} \wedge \omega_{\alpha_{r}}^{\gamma}+\widetilde{\Omega}_{\alpha_{r}}^{\beta} \\
& \equiv 0\left(\bmod \omega^{\beta}, \omega_{i}^{\beta}, \omega_{\alpha_{1}}^{\beta}, \ldots, \omega_{\alpha_{\nu-1}}^{\beta}\right), \text { where } \gamma \geqslant\left(\begin{array}{c}
\dot{n}+\nu \\
\nu
\end{array}\right) .
\end{aligned}
$$

Therefore it is seen that the distribution $\mathfrak{P}$ is completely integrable. 
For any point $x$ in $M_{n}(c)$, let $M(x)$ be the maximal integral submanifold of $\mathfrak{M}\}$ through $x$. Then $M(x)$ is of $\left\{\left(\begin{array}{c}n+\nu \\ \nu\end{array}\right)-1\right\}$-dimensional and by the construction it is totally geodesic in $M_{n+p}(\tilde{c})$. Thus there exists an $\left\{\left(\begin{array}{c}n+\nu \\ \nu\end{array}\right)-1\right\}$-dimensional totally geodesic submanifold of $M_{n+p}(\tilde{c})$ in which the given submanifold $M_{n}(c)$ is immersed. This is a contradiction to the assumption that the immersion is full.

This completes the proof.

\section{BIBLIOGRAPHY}

1. E. Calabi, Isometric imbedding of complex manifolds, Ann. of Math. (2) 58 (1953), 1-23. MR 15, 160.

2. K. Ogiue, On Kaehler immersions, Canad. J. Math. 24 (1972), 1178-1182. MR 47 \#7663.

3. n-dimensional complex space forms immersed in $\{n+n(n+1) / 2\}$-dimensional complex space forms, J. Math. Soc. Japan 24 (1972), 518-526. MR 46 \#907.

4. Differential geometry of Kaehler submanifolds, Advances in Math. 13 (1974), 73-114. MR 49 \#11444.

DEPARTMENT OF MATHEMATICS, TOKYO UNIVERSITY OF AGRICULTURE AND TECHNOLOGY, TOKYO, JAPAN (Current address of Hisao Nakagawa)

DEPARTMENT OF MATHEMATICS, MICHIGAN STATE UNIVERSITY, EAST LANSING, MICHIGAN 48823

Current address (K. Ogiue): Department of Mathematics, Tokyo Metropolitan University, Tokyo, Japan 